\title{
Metodologias alternativas no ensino de fisiologia humana: um relato de vivência no ensino superior
}

\author{
Anthony Marcos Gomes dos Santos
}

Elayne Cristine Soares da Silva

\section{Resumo}

Este relato de vivência é destinado aos docentes do ensino médio e superior, pois retrata a experiência com 40 alunos do $3^{\circ}$ período da Licenciatura Plena em Ciências Biológicas, na disciplina de Fisiologia Humana Básica. Os objetivos foram avaliar a eficácia e o uso de métodos alternativos no processo de ensino em Fisiologia Humana; bem como avaliar a utilização de textos acadêmicos como facilitadores na aquisição da linguagem acadêmica e aplicabilidade dos conceitos vistos na disciplina, correlacionando os assuntos com temas do dia a dia. Para isso, foram utilizadas as seguintes metodologias: Team Based Learning ou Aprendizagem em Times, Ensino por imersão, Aprendizagem por pares, Aprendizagem Baseada em Jogos. Como resultados foi possível dinamizar as aulas, promovendo o ensino ativo de Fisiologia Humana no ensino superior, facilitando os processos de ensino e aprendizagem, além de promover a compreensão entre a diferença entre o ensino tradicional e o ensino ativo da Fisiologia Humana no ensino superior, permitindo o refinamento das práticas pedagógicas a serem utilizadas no futuro e trazendo subsídios à prática sobre como essas intervenções pedagógicas trabalhos.

Palavras-chave: Fisiologia Humana. Metodologias ativas. Jogos didáticos. 


\title{
Alternative methodologies in human physiology teaching: an experience report in the superior education
}

\author{
Anthony Marcos Gomes dos Santos \\ Elayne Cristine Soares da Silva
}

\section{Abstract}

This experience report is intended to the teachers of middle and superior education, cause shares the experience with 40 students of the 3 rd period of Full degree in Biologic Science, in the Basic Human Physiology discipline. The objectives were to evaluate the effectiveness and the use of alternative methods in the teaching process of Human Physiology; as well as assessing the utilization of academic texts as facilitators in the acquisition of academic language and applicability of concepts seen in the course of discipline, correlating subjects with day by day themes. For this, was utilized the followings methodologies: Team-Based Learning, Immersion Teaching, Peer Instruction, Games Based Learning. As a result, it was possible to streamline classes, promoting active teaching of Human Physiology in higher education, through pedagogical practices that facilitated the teaching processes, motivating learning, facilitating the processes of teaching and learning, besides promoting the comprehension between the difference of traditional teaching and active teaching of Human Physiology in the superior education, allowing the refinement os pedagogic practices to be used in the future and bringing practice subsidies on how these pedagogical interventions work.

Keywords: Human Physiology. Actives Methodologies.; Didactic Games. 


\section{Introdução}

A Fisiologia Humana (FH) é um dos componentes curriculares mais importantes para cursos da área de saúde e até mesmo no ensino médio, dentro das disciplinas de Ciências e Biologia (MALAFAIA, 2010; BERG, 2012; BORGES \& MELLO-CARPES, 2015). Segundo Silverthorn (2010), a Fisiologia é o estudo do funcionamento normal de um organismo vivo e de suas partes componentes, incluindo todos os seus processos físicos e químicos. A Fisiologia também aparece como base para o conhecimento do próprio corpo, trazendo compreensão sobre suas atividades, correlacionando com alterações do dia a dia (TORTORA e DIRRICKSON, 2016).

Os conceitos de Fisiologia estão presentes em diferentes disciplinas tanto no ensino superior (Anatomia, Parasitologia, Epidemiologia, Farmacologia), quanto no ensino médio (Química, Educação física, Educação ambiental) (REZENDE, 2013; BORGES et al. 2016). A FH desempenha um papel importante na aquisição de conceitos, processos e até mesmo de uma linguagem específica acerca de processos biológicos fundamentais para manutenção da vida em diversas biociências (BORGES et al. 2016). Devido ao fato desses conceitos se estenderem a diversos campos como inovação terapêutica, qualidade de vida e desenvolvimento tecnológico, é necessário que se desenvolva uma visão contextualizada e integrada não só dos seres humanos, mas dos diversos seres vivos, compreendendo assim como os organismos funcionam como um todo (VANZELA, 2013).

Campos e colaboradores (2003) falam sobre a importância de uma formação crítica e contextualizada, a qual permite que um largo conhecimento sobre o funcionamento do corpo humano, seja um domínio relevante na manutenção do bem-estar e da saúde. Nesta última, a a fisiologia é base para o reconhecimento de situações patológicas, auxiliando no diagnóstico e no entendimento em estudos científicos avançados (TORTORA, 2006).

Diversos fatores tornam difícil os processos de aprendizagem da Fisiologia Humana, em diferentes níveis (REZENDE, 2013; VANZELA, 2013). Considera-se que o maior problema na sua compreensão é a fragmentação dos conteúdos, já que os tópicos são vistos de maneira isoladas e sem contextualização (MORIN, 2002; LENGERT E MARCHESE, 2007; LARA, 2014). Essa fragmentação dificulta que os alunos relacionem um conteúdo com o outro, distanciando da realidade profissional e da sociedade (VARGAS, 2001; ALMEIDA e CARVALHO, 2002). O afastamento do conteúdo da realidade socio-histórica-cultural e econômica dos alunos também dificulta o processo (SOARES, 2001; VANZELA, 2013).

Borges e colaboradores (2016) citam uma série de outros fatores que dificultam o fortalecimento de uma aprendizagem concreta e significativa na Fisiologia Humana. Segundo eles, estudantes enfrentam questões de motivação provocadas por fatores como as deficiências prévias de formação; infraestrutura escolar inadequada; professores com qualificação deficiente; diferentes interesses pessoais; modelo de ensino-aprendizagem excessivamente 
concentrado no professor; foco na memorização de conteúdos; segmentação do conhecimento em disciplinas isoladas; abordagens baseadas em aulas expositivas.

As necessidades emergentes da sociedade e da educação em seus diferentes graus, têm trazido os holofotes para a prática docente e como seus resultados interferem na formação de cada indivíduo, na sua prática profissional (CARVALHO e NOVO, 2005; MELO e DAMASCENO, 2006; FONAZIERO et al., 2010). No entanto, existem outras variáveis envolvidas, pois, embora os professores saibam das dificuldades acerca do ensino e aprendizagem de $\mathrm{FH}$, há resistência à mudança deste cenário, como a fragmentação curricular e o método tradicional ainda majoritário no ensino (ALTERMANN, 2016). Faz-se necessário, o incentivo ao desenvolvimento da autonomia e da criticidade que conduzam o aluno na construção de um conhecimento sólido (BRASIL, 1999; CYRINO E TORALLES-PEREIRA, 2004; CRUZ, 2010; VANZELA, 2013).

Borges e colaboradores (2016) ressaltam a importância da motivação nesse processo de evolução da práxis pedagógica (contextualização de conteúdo, desenvolvimento de atividades práticas como complemento da teoria, fornecimento de subsídio teórico a ser aplicado em outras áreas do conhecimento e capacidade de aplicação das diferentes temáticas da fisiologia na sociedade) na $\mathrm{FH}$ e aponta fatores a serem analisados pelos professores que podem ajudar a despertar, nos alunos, o interesse pelo estudo nessa disciplina. Eles afirmam que devemos analisar ferramentas e materiais que podem ser utilizados nos processos de ensino e aprendizagem; refletir sobre as diferentes abordagens pedagógicas escolhendo as que aumentam a retenção do conhecimento, discutir e fortalecer organizações curriculares mais integradas, interdisciplinares e que abordem a prática profissional e a realidade biopsicossocial, histórica e econômica dos alunos.

\section{Objetivos}

Avaliar a eficácia e o uso de métodos alternativos no processo de ensino e aprendizagem em Fisiologia Humana; avaliar a utilização de textos acadêmicos, como facilitadores na aquisição da linguagem acadêmica e aplicabilidade dos conceitos vistos na disciplina de Fisiologia Humana; relacionar situações do dia-dia aos assuntos trabalhados em sala de aula.

\section{O papel das metodologias ativas}

O método tradicional, por não facilitar a contextualização, ludicidade e autonomia dos alunos é um dos fatores que elevam a evasão, e reprovação na disciplina de Fisiologia Humana, além de que se mal aplicado (do ponto de vista de não construir todas as competência teóricas e práticas que a disciplina requer e propõe) pode acabar formando um profissional despreparado para atuar na sociedade (LACERDA, 2018). Diversos aspectos sociais, cognitivos 
e afetivos são importantes no processo de ensino (MASETTO, 2014). Métodos de ensino não tradicionais como as metodologias ativas surgem como uma resposta a problemática trazida anteriormente. Quando bem estruturados e elaborados auxiliam na formação integral do indivíduo não só pedagogicamente, mas agregando competências cognitivas e promovendo uma melhor formação profissional (LACERDA, 2018).

Nas metodologias ativas, o professor assume um papel de mediador dos processos de ensino e aprendizagem enquanto o aluno é o protagonista. O professor instiga o aluno para que ele possa desenvolver a vontade de buscar sobre o tema, reconstroem as experiências vividas anteriormente a partir do novo conhecimento adquirido, tem sua bagagem sociocultural valorizada, estimula o trabalho em grupo, respeita o tempo singular de aprendizado dentre outros benefícios (WALL, 2008). Miltre (2008) afirma que as metodologias ativas formam novos conceitos e atitudes que ampliam o conhecimento, pautados em críticas, debates, e protagonismo do aluno.

Moran (2018) diz que a motivação e a proximidade dos alunos com o conteúdo tornam a aprendizagem significativa. O diálogo sobre as atividades e a forma de realizá-las também fortalecem esse processo. Essa aprendizagem significativa fortalece o desenvolvimento intelectual do indivíduo em toda sua extensão cognitiva, facilitando a capacidade de comparar, criticar, justificar, argumentar, entender processos, buscar e filtrar informações, criar, inventar, imaginar; além de facilitar a organização, produção e compartilhamento de conhecimento; sendo mais fácil reproduzi-los de diferentes formas, quando necessário (AUSUBEL, 1966; MASETTO, 2010).

É importante que as metodologias aplicadas consigam abordar os conteúdos de interesse, ao mesmo tempo em que guiam para a construção do profissional ou indivíduo que se deseja alcançar (MORAN, 2018). As metodologias ativas são bastante diversas e permitem que o professor possa escolher qual vai aplicar, levando em consideração tamanho da turma, recursos financeiros, proposta da atividade, além de interesses e preferências dos alunos (ROCHA, 2014). Podem ser citadas entre as mais utilizadas, a Aprendizagem Baseada em Problemas, que consiste na necessidade do aluno resolver um problema proposto pelo professor, relacionado com o assunto que se quer abordar e normalmente presente no dia a dia do estudante (GUERRA, 2014); Aprendizagem Baseada em Projetos, que consiste na resolução colaborativa de um problema, processo mal estruturado ou proposta de intervenção prática, seja de pesquisa, criação de produto, objeto. É bastante interdisciplinar e aplicável a realidade profissional (BENDER, 2015); o Estudo de Caso quando os alunos analisam um caso real, em toda sua amplitude e complexidade, e propõem uma solução interdisciplinar que envolva o conteúdo visto na disciplina (YN, 2015).

Além dessas, há a Aprendizagem Baseada em Times, que favorece ao aluno a aplicação de um conteúdo visto e aprenda também sobre a aplicabilidade social, científica, tecnológica; 
dentro deste contexto trabalhado (MICHAELSEN E SWEET, 2008); As Simulações que permitem o fortalecimento do que foi visto na aula expositiva (MARINS et al., 2008); Aprendizagem por Pares, que consiste que os alunos fortaleçam o aprendizado sobre algum tópico discutindo entre si (LASRY et al., 2008); Ensino Híbrido, que introduz as tecnologias digitais de informação e comunicação tanto nas abordagens pedagógicas quanto na avaliação da aprendizagem (VALENTE, 2014); Sala de Aula Invertida, onde todo o conteúdo referente a aula é previamente passado para os alunos, que vem para sala de aula tirar dúvidas e trabalhar os conteúdos previamente estudados (VALENTE, 2014).

Ainda pode-se incluir nessas metodologias ativas a Aprendizagem Baseada em Jogos, que consiste na utilização desses durante todo os processos de ensino e aprendizagem, desde a elucidação de conteúdos até mesmo a avaliação da aprendizagem (ROLAND, 2010). Os jogos proporcionam um ambiente divertido, atrativo e dinâmico de aprendizagem, estimulando a espontaneidade enquanto promove a aquisição de um determinado conteúdo (VARGAS, 2009). É necessário que sejam construídos e configurados para que possam auxiliar da forma que desejar o professor, atingindo assim os objetivos propostos (NETO, 2013).

Existem diversos tipos de jogos como ação, cassino, aventura, role-Playing games (RPG), lógicos, estratégicos, aventura, entre outros; que são classificados de acordo com a sua funcionalidade e objetivo (TAROUCO, 2004). O jogo estimula a imaginação, criatividade, tomada de decisões, atenção, concentração, curiosidade e aumenta a atenção do aluno por muito tempo em determinada da atividade (FORTUNA, 2003). A prática de jogar alia a cognição, a ludicidade, enriquece a aprendizagem, permitindo a visualização e acomodação de conceitos abstratos, ao mesmo tempo que favorece a motivação interna e a aquisição da linguagem (CAMPOS et al., 2003).

Baseando-se no que foi exposto, espera-se com este trabalho, reforçar a utilização de metodologias ativas como facilitadoras na sala de aula, visto que as mesmas, segundo a literatura, estimulam as relações interpessoais, o auto aprendizado, a aquisição da linguagem, interpretação de fatos, organização de ideias, criação de hipóteses, comparação e aplicação de fatos a problemas concretos (BERBEL, 2011; SOUZA et al., 2014; MORAN, 2018).

\section{Métodos}

A intervenção foi desenvolvida no âmbito da Disciplina de Fisiologia Humana Básica, no terceiro período do Curso de Licenciatura Plena em Ciências Biológicas de uma universidade pública federal no ano de 2018. A disciplina contou com o auxílio de dois monitores do programa de monitoria do departamento ofertante da disciplina, sendo eles alunos do $7^{\circ}$ e $8^{\circ}$ período também de Licenciatura Plena em Ciências Biológicas. Os monitores junto com a professora responsável participaram do processo de elaboração, aplicação e avaliação das metodologias. A atividade passou por diversas etapas, desde a seleção dos 
conteúdos para aplicação das metodologias ativas, elaboração das propostas, construção do material a ser utilizado, aplicação em sala de aula e avaliação do impacto da das metodologias ativas no processo pedagógico.

A turma participante do processo era do turno noturno e tinha em média 40 alunos, muitos deles repetentes. As aulas aconteciam dois dias na semana, tendo cada encontro a duração de duas horas, totalizando 60 horas, durante o período de um semestre. Além do espaço formal da sala de aula, os alunos tinham a disposição o auxílio dos monitores em três dias diferentes da semana, com o laboratório de aulas práticas a disposição para os encontros. O horário e a periodicidade dos momentos da monitoria ficavam à disposição dos alunos.

Em relação aos espaços, além da sala de aula e do laboratório de aulas práticas, incentivou-se a participação no Ambiente Virtual de Aprendizagem (AVA), para melhor compartilhamento e comunicação entre a turma, professora e monitores. Materiais como data show, cartolina, marcadores para quadro branco, pinceis atômicos, lápis hidrocor e folhas de papel ofício; além de tensiômetros, estetoscópios, glicosímetros e utensílios laboratoriais como ellermeyer, tubos de ensaio, beckers, banho maria e cubetas, também foram utilizados.

Na primeira etapa do processo de intervenção foi utilizada a metodologia Team Based Learning ou Aprendizagem em Times. Os alunos foram divididos em oito grupos, cada um com cinco participantes e tiveram que aplicar os conhecimentos vistos na disciplina (como exemplo, fisiologia nervosa e endócrina), na interpretação de artigos científicos, sendo capazes de reconhecer processos, termos, funções, substâncias e a aplicabilidade do conteúdo estudado de diversas formas. Os artigos abordavam temas desde vias clássicas da fisiologia, como processos fisiopatológicos de doenças. Para isso, contaram com o AVA e a monitoria para guiálos na interpretação e preparação de uma apresentação do artigo, que seria o momento de compartilhamento com todos demais alunos da turma.

Além disso, o Ensino por imersão foi utilizado em busca de um aprendizado prático, o qual aconteceu por meio de atividades experimentais no laboratório de aulas práticas. Os alunos puderam se familiarizar com o ambiente laboratorial e regras de biossegurança durante as aulas; recebendo roteiros que ensinavam sobre a preparação e montagem dos experimentos. Em seguida, tiveram que organizar, tabelar e interpretar os dados colhidos, embasando os mesmos com a teoria vista em sala de aula e justificando os resultados a partir de livros e artigos científicos.

A Aprendizagem por pares aconteceu nos momentos da monitoria. $\mathrm{O}$ atendimento era individual e os monitores, sempre que possível utilizavam uma abordagem diferente para cada aluno. Os discentes aproveitavam esse momento extraclasse para tirar dúvidas, responder exercícios e compartilhar suas descobertas, embasar teoricamente as observações do dia a dia e correlacionar as mesmas com a Fisiologia Humana. 
A eficácia dessas abordagens foi verificada na primeira nota, por meio do somatório das pontuações obtidas na prova teórica (Com pontuação máxima 7,0. Incluia questões abertas e de múltipla escolha, baseada nos conteúdos vistos de diversas formas), relatório de aulas práticas no laboratório (Com pontuação máxima 1,o) e apresentação do artigo em grupo (Com pontuação máxima 2,0). A composição das notas foi repassada aos alunos após correção, sendo feita também discussão das questões de prova, juntamente com a professora, permitindo aos alunos analisarem seus erros.

Para composição da segunda nota foi feita a prova teórica, com os mesmos critérios citados na primeira nota; sendo acrescentada uma atividade de Aprendizagem Baseada em Jogos (Com pontuação máxima 3,0). Os alunos foram apresentados a metodologia e foram divididos em oito grupos com cinco participantes. Cada grupo com um tema específico abordado na disciplina e sorteado pela professora (como exemplo, fisiologia cardiovascular, fisiologia digestiva, fisiologia renal). Os alunos tiveram a tarefa de estudar sobre o assunto, elaborar e desenvolver jogos relacionados ao seu conteúdo com grau de dificuldade voltado para ensino médio, visto que são alunos de licenciatura. Os monitores estiveram disponíveis para orientar na construção dos jogos, sob avaliação da docente responsável.

Além disso, a fim de familiarizar os alunos com a escrita científica e a aquisição da linguagem específica, os alunos tiveram que escrever um relatório científico contendo uma breve revisão de literatura, justificava, materiais e métodos utilizados na construção do seu jogo, juntamente com a forma de utilizá-lo. Os alunos também receberam orientação sobre como escrever o relatório técnico. Os melhores foram encaminhados para um congresso local, mediante avaliação da professora responsável pela disciplina, monitores e outra professora convidada. Os jogos foram testados por alunos da turma, professora e monitores, sendo avaliada a aplicabilidade, abordagem do tema, acabamento e fidelidade ao grau e tema proposto.

\section{Reflexões/análise prática}

Através do uso integrativo de várias metodologias de ensino, foi possível observar uma maior participação e interesse dos estudantes, os quais muitas vezes já chegavam cansados da rotina diária de trabalho, lembrando que se tratava de um curso noturno. A maior dificuldade do docente, seria tornar a aula mais atrativa e dinâmica. Como uma forma de incentivo, a avaliação foi feita de forma conjunta (atividades e prova).

Em relação as atividades, as pontuações foram dadas com base na participação, execução correta da proposta, veracidade das informações trazidas pelos discentes e correção do conteúdo específico. Quanto a prova teórica, a pontuação foi dada de acordo com o número de respostas certas. Com isso, distribuiu-se os pesos da nota, estimulando a leitura e participação contínua dos discentes. Apenas uma aluna, optou por não participar das 
atividades em grupo. Isso é uma limitação que pode ser vivenciada em qualquer metodologia que envolva o trabalho em grupo. Neste caso, a participação não foi forçada e forma de avaliação foi feita individual.

Embora havendo tempo, disponibilidade de espaço físico e monitores, os momentos de monitoria não eram utilizados por muitos, tendo uma média de 3-5 frequentadores por semana, o que representou aproximadamente $10 \%$ da turma. Isso também pode refletir a questão de que alguns trabalhavam nos horários sem aula, participavam de outras atividades acadêmicas ou não tinham o hábito/interesse em receber esse tipo de orientação.

Essa questão de disponibilidade dos alunos, especialmente do turno noturno, abre margem para uma discussão acerca de currículo, estruturação pedagógica e o desenvolvimento de propostas que permitam a inclusão desses alunos nos demais ambientes que a universidade proporciona, além da sala de aula. As atividades lúdicas, metodologias ativas e qualquer outra ação fora do padrão tradicional da sala de aula podem ser ferramentas no combate ao cansaço e desestímulo desses alunos, causados pelos fatores citados acima.

Fruto do trabalho desenvolvido em sala de aula, os jogos elaborados pelos discentes foram um momento de descontração e participação da maioria da turma; além de serem encaminhados a um congresso local; permitindo a presença deles em atividades vinculadas a disciplina fora da sala de aula. Alguns inclusive, nunca tinham participado de um congresso ou enviado algum trabalho neste sentido. Da mesma forma, a interpretação de artigos científicos também foi algo novo para alguns deles, permitindo identificar conteúdo das aulas em temas abordados com aplicabilidade.

É importante ressaltar que cada professor pode utilizar de vários recursos para a finalidade maior que é o aprendizado dos discentes. Lembrando que cada vez mais tem sido difícil mantê-los interessados e participativos, apenas com os métodos tradicionais de ensino. É aí que as metodologias ativas podem ser aliadas nesta missão.

\section{Referências}

ALMEIDA, M. da C. de; CARVALHO, E. de A.; MORIN, E. Educação e complexidade: os sete saberes e outros ensaios. 2002.

ALTERMANN, C.; NEVES, Ben-Hur S.; MELLO-CARPES, P B. The inclusion of undergraduate students in physiology outreach activities improves their physiology learning and understanding skills. Advances in physiology education, v. 40, n. 4, p. 529-532, 2016.

AUSUBEL, D P. Meaningful reception learning and the acquisition of concepts. In: Analyses of concept learning. Academic press, 1966. p. 157-175.

BENDER, W N. Aprendizagem baseada em projetos: educação diferenciada para o século XXI. Penso Editora, 2015.

ISSN 2526-2882 
BERBEL, N. A. N.. As metodologias ativas e a promoção da autonomia de estudantes. Semina: Ciências Sociais e Humanas, v. 32, n. 1, p. 25-40, 2011.

BERG, R. MG. "Physiological curiosity of the week": a teaching tool to facilitate self-directed learning and student participation during a cardiovascular physiology course. Advances in physiology education, v. 36, n. 4, p. 356-357, 2012.

BORGES, G. A. et al. Body: Um jogo digital educacional de tabuleiro na área de fisiologia humana. Proceedings of SBGames, p. 412-420, 2016.

BORGES, S; MELLO-CARPES, P. B. Undergraduate students as promoters of science dissemination: a strategy to increase students' interest in physiology. Advances in physiology education, v. 39, n. 2, p. 133-136, 2015.

BRASIL, M. E. C. Secretaria de Educação média e Tecnológica. Parâmetros Curriculares Nacionais: Ensino Médio. Brasília, 1999.

CAMPOS, L. M. L.; BORTOLOTO, T. M.; FELÍCIO, A. K. C. A produção de jogos didáticos para o ensino de ciências e biologia: uma proposta para favorecer a aprendizagem. Caderno dos núcleos de Ensino, v. 3548, 2003.

CARVAlHO, F. A. H. de; NOVO, M. S.. Aprender como aprender: otimização da aprendizagem. 2005 .

CRUZ, S. R. R.. Professores e mídias digitais: em busca da integração às práticas pedagógicas. Diálogos Educacionais em Revista, v. 1, n. 1, 2010.

CYRINO, E. G.; TORALLES-PEREIRA, M. L.. Discovery-based teaching and learning strategies in health: problematization and problem-based learning. Cadernos de saúde pública, v. 20, n. 3, p. 780-788, 2004

ROSA, L. S. da et al. Difusão da fisiologia através da capacitação de docentes da Educação Básica. Revista Ciência em Extensão, v. 9, n. 2, p. 128-140, 2013.

SILVA NETO, S. R. da et al. Jogos educacionais como ferramenta de auxílio em sala de aula. In: Anais do Workshop de Informática na Escola. 2013. p. 130.

FORNAZIERO, C. C. et al. O ensino da anatomia: integração do corpo humano e meio ambiente. Rev Bras Educ Med, v. 34, n. 2, p. 290-7, 2010.

FORTUNA, T. R.. Sala de aula é lugar de brincar. Planejamento em destaque: análises menos convencionais. Porto Alegre: Mediação, p. 147-164, 2000.

GUERRA, A. Problem based learning and sustainable engineering education: challenges for 21st century. Department of Development and Planning, Aalborg University. http://vbn. aau. dk/files/198754491/PhD_monograph_vol. _I_. pdf. Acesso em o 7 fev. 2019., v. 18, p.

LARA, M. V. et al. Objetos de aprendizagem como coadjuvantes do processo de ensinoaprendizagem de Fisiologia humana. Revista de Ensino em Bioquímica, v. 12, n. 1, p. 34-47, 2014 
LASRY, N.. Clickers or flashcards: Is there really a difference?. The Physics Teacher, v. 46, n. 4, p. 242-244, 2008.

LENGERT, J. A. M. V. H.; MARCHESE, M. C. A utilização de um tema atual - o estresse como elemento 78 motivador e integrador para o estudo da fisiologia humana no ensino médio: a percepção dos alunos sobre o seu estresse - causas, consequências e controle. Secretaria de Educação do Paraná, 2007. Disponível em: http://www.diaadiaeducacao.pr.gov.br/portals/cadernospde/pdebusca/producoes_ pde/2012/2012_ufpr_cien_pdp_josefa_jesus_do_nascimento.pdf. Acesso em: o7 fev. 2019.

MALAFAIA, G.; BÁRBARA, V. F.; LIMA RODRIGUES, A. S.i de. Análise das concepções e opiniões de discentes sobre o ensino da biologia. Revista Eletrônica de Educação, v. 4, n. 2, p. 165-182, 2010.

MASETTO, M.. Docência na universidade. Papirus Editora, 2014.

MELO, F. N. de P.; DAMASCENO, M. M. C.. A construção de um software educativo sobre ausculta dos sons respiratórios. Revista da Escola de Enfermagem da USP, v. 40, n. 4, p. 563-569, 2006.

MICHAELSEN, L. K.; SWEET, M.. The essential elements of team-based learning. New directions for teaching and learning, v. 2008, n. 116, p. 7-27, 2008.

MITRE, S. M. et al. Metodologias ativas de ensino-aprendizagem na formação profissional em saúde: debates atuais. Ciência \& saúde coletiva, v. 13, p. 2133-2144, 2008.

MORAN, J.. Metodologias ativas para uma aprendizagem mais profunda. Metodologias ativas para uma educação inovadora: uma abordagem teórico-prática. Porto Alegre: Penso, p. 02-25, 2018.

MORIN, E.. Educação e complexidade: os sete saberes e outros ensaios. In: Educação e complexidade: os sete saberes e outros ensaios. 2002.

PEDROSO, C. V.. Jogos didáticos no ensino de biologia: uma proposta metodológica baseada em módulo didático. In: ANALES DE IX CONGRESSO NACIONAL DE EDUCAÇÃO (EDUCERE) \& III ENCONTRO SUL BRASILEIRO DE PSICOPEDAGOGIA. 2009. p. 3182-3190.

REZENDE, I. M. N. de; SILVA COUTINHO, A. da; ARAÚJO, Monica Lopes Folena. Educação Ambiental e Fisiologia Humana: compreensões e práticas de professores de biologia. Alexandria: Revista de Educação em Ciência e Tecnologia, v. 6, n. 3, p. 211-226, 2013.

ROCHA, H. M.; LEMOS, W. de M.. Metodologias ativas: do que estamos falando? Base conceitual e relato de pesquisa em andamento. IX Simpósio Pedagógico e Pesquisas em Comunicação. Resende, Brasil: Associação Educacional Dom Boston, p. 12, 2014.

ROLAND, L. C. et al. Jogos educacionais. RENOTE, v. 2, n. 1, 2010. 
SANTOS, L. M. dos; LACERDA, F. C. B.. Integralidade na formação do ensino superior: metodologias ativas de aprendizagem. Avaliação: Revista da Avaliação da Educação Superior, v. 23, n. 3, 2018.

SILVERTHORN, D. U.. Fisiologia humana: uma abordagem integrada. Artmed editora, 2010.

SOARES, Magda. As pesquisas nas áreas específicas influenciando o curso de formação de professores. O papel da pesquisa na formação e na prática dos professores, v. 3, p. 91106, 2001.

SOUZA, C. da S.; IGLESIAS, A. G.; PAZIN-FILHO, A.. Estratégias inovadoras para métodos de ensino tradicionais: aspectos gerais. Medicina (Ribeirão Preto), p. 284-292, 2014.

TAROUCO, L. M. R. et al. Jogos educacionais. RENOTE: revista novas tecnologias na educação [recurso eletrônico]. Porto Alegre, RS, 2004.

TORTORA, G. J. GRABOWSKI, S. R.. Princípios de anatomia e fisiologia. Rio de Janeiro: Guanabara Koogan, 2006.

TORTORA, G. J.; DERRICKSON, B.. Corpo Humano-: Fundamentos de Anatomia e Fisiologia. Artmed Editora, 2016.

VALENTE, J. A.. Aprendizagem Ativa no Ensino Superior: a proposta da sala de aula invertida. Puc. São Paulo, 2014.

VANZELA, E. C.; BALBO, S. L.; DELLA JUSTINA, L. A.. A integração dos sistemas fisiológicos e sua compreensão por alunos do nível médio. Arquivos do Museu Dinâmico Interdisciplinar, v. 11, n. 3, p. 12-19, 2013.

VARGAS, L. H. M.. A bioquímica e a aprendizagem baseada em problemas. Revista de Ensino de Bioquímica, v. 1, n. 1, p. 15-19, 2001.

WALL, M. L.; PRADO, ML do; CARRARO, T. E. A experiência de realizar um Estágio Docência aplicando metodologias ativas. Acta Paul Enferm, v. 21, n. 3, p. 515-9, 2008.

YIN, R. K. Estudo de Caso-: Planejamento e Métodos. Bookman editora, 2015.

\section{Biografia Resumida}

Anthony Marcos Gomes dos Santos: Licenciado em Ciências Biológicas pela Universidade Federal Rural de Pernambuco. Tem experiência no desenvolvimento, aplicação e avaliação de metodologias ativas no ensino de morfologia e fisiologia humana, educação multicultural e no desenvolvimento de atividades práticas para o ensino fundamental e superior. Mestrando em Biociência Animal, trabalha principalmente com histofisiologia de doenças crônicas.

Lattes: http://lattes.cnpq.br/4572948318160798

ISSN 2526-2882 
Contato: anthonymarcos20@gmail.com

Elayne Cristine Soares da Silva: Licenciada em Ciências (Habilitação Biologia) pela Universidade de Pernambuco; Graduada em Medicina Veterinária pela Universidade Federal Rural de Pernambuco; Especialista em Educação Ambiental pela Universidade de Pernambuco; Mestre em Ciência Veterinária pela Universidade Federal Rural de Pernambuco; Doutora em Biotecnologia pela Rede Nordeste de Biotecnologia (Universidade do Ceará). Professora Adjunto A II da Área de Fisiologia e Farmacologia, Departamento de Morfologia e Fisiologia Animal, Universidade Federal Rural de Pernambuco. Trabalha com oncologia experimental, fisiologia e farmacologia.

Lattes: http://lattes.cnpq.br/2064359935012193

Contato:la_cristine@yahoo.com.br, elayne.silva@ufrpe.br 